\title{
Modelo para Implantação de Custeio por Características
}

Tiago Pascoal Filomena

Mestrado em Engenharia de Produção pela Universidade Federal do Rio Grande do

Sul - UFRGS

Professor da Pontifícia Universidade Católica do Rio Grande do Sul - PUC/RS

Praça Argentina 9. 20 andar/LOPP. Porto Alegre/RS. CEP: 90040-020

E-mail: tiagofilomena@gmail.com

Francisco José Kliemann Neto

Doutorado em Engenharia de Produção - Institut National Polytechnique (França)

Professor da Universidade Federal do Rio Grande do Sul - UFRGS

Av. Osvaldo Aranha, 99, 5 andar. (UFRGS/DEPT). Porto Alegre/RS. CEP: 90035-190

E-mail:kliemann@producao.ufrgs.br

\section{RESUMO}

Este estudo apresenta um modelo para implementação do custeio por características. O custeio por características (feature costing) é um tema emergente em gestão de custos. Não se trata de um método de custeio propriamente dito, e sim o que passa a ser repensado é o objeto de custeio, deixando de ser o produto e passando a ser as características do produto. $\mathrm{O}$ método do custo-padrão, o método do custeio baseado em atividades ( $A B C$ ) e o método da unidade de esforço de produção (UEP) são utilizados para a operacionalização do modelo. O modelo é aplicado em um grupo de poltronas de uma indústria fabricante de carrocerias de ônibus.

Palavras-chave: Custeio por características. Custeio baseado em atividades. Unidade de esforço de produção. UEP.

\section{Model for Feature Based-Costing Implementation}

\section{ABSTRACT}

This paper presents a model to implement featured-based cost management system. Feature costing is an emerging topic in cost management. Feature costing is not a cost method itself; it is a proposition to change the cost object from product to features. To operationalize the feature costing it was used the standard costing, the activity-based costing (ABC) and the unit of production effort methods. This model was applied to a group of seat of a body bus manufacturing company.

Key words: Feature costing. Activity-based costing. Unit of production effort. 


\section{INTRODUÇÃO}

A evolução da administração da produção vem demandando uma reestruturação dos sistemas de custeio. Até 1925, quase todas as práticas de gestão de custos tradicionais - cálculo do custo de mão-de-obra, matéria-prima e despesas gerais - já haviam sido desenvolvidas. No entanto, a evolução tímida da tecnologia de informação e a pressão do mercado por balanços financeiros auditados fez com que os sistemas de custeio ficassem estagnados, mesmo com a evolução da administração da produção (Johnson e Kaplan, 1993).

Com o avanço da tecnologia de informação na década de 1960, era provável que o desenvolvimento dos sistemas de custeio fosse alavancado. Contudo, a simplificação contábil continuou imperando e o que se viu foi a melhoria da administração de custos baseada na separação de custos fixos e variáveis, análise esta eficiente em uma empresa monoprodutora, porém pouco útil para o cenário de produtos diversificados que começava a surgir na época. A fragilidade do método de separação de custos fixos e variáveis se mostra na medida em que este nem mesmo questiona a origem e a taxa de consumo dos custos fixos pelos produtos (Johnson e Kaplan, 1993).

O surgimento de tecnologias avançadas - robótica, projeto auxiliado por computador ( $C A D)$, sistemas flexíveis de manufatura - mudaram os processos industriais, modificando, assim, o perfil de custos das empresas. Essas mudanças resultaram em taxas de custos indiretos cada vez mais elevadas, afetando a base sobre a qual os custos eram alocados, ou seja, a mão-de-obra. Com isso, as décadas de 1980 e 1990 trouxeram inovações significativas para a gestão de custos, a mais importante, talvez, seja o custeio baseado em atividades ( $A B C$ ), método eficiente para alocação de custos indiretos (Berliner e Brimson, 1988).

Atualmente, outro tema vem emergindo na área de gestão de custos: o custeio por características (feature costing). Trabalhos como os de Ou-Yang e Lin (1997), Leibl et al. (1999), Rosa e Lunkes (2006), Filomena et al. (2009) mas, principalmente, o realizado por Brimson $(1998,2001)$ apresentam alguns estudos referentes ao tema. 
O custeio por características não é um método de custeio propriamente dito. Brimson (1998) apresenta o custeio por características como sendo um desdobramento do custeio por atividades. Na verdade, o que passa a ser repensado no custeio por características é o objeto de custeio, deixando de ser o produto e passando a ser as características do produto.

Brimson (1998) assinala que apesar das melhorias trazidas pelo custeio ABC aos sistemas de gestão de custos, a permanência do $A B C$ como sistema único de gestão de custos é questionável. Isso se dá por duas razões: (i) quanto mais detalhado o sistema de custeio, mais útil para a equipe operacional, porém mais difícil é a coleta de dados para a manutenção do sistema; logo, o número de atividades mapeadas no sistema diminui; (ii) A diminuição do número de atividades faz com que o sistema deixe de ser usado pela equipe operacional, e sua relevância passa a ser somente gerencial.

As características dos produtos são críticas para vendas, marketing, pesquisa e desenvolvimento (P\&D) e fabricação. Marketing deve saber as características dos produtos que mais satisfazem o cliente, e P\&D deve desenvolver produtos e processos com custos adequados às características necessárias. As características estão relacionadas aos fatores que causam variação de custos dos processos; logo o método pode criar um melhor entendimento para diminuição do custo do produto (Brimson, 1998).

As etapas propostas por Brimson (1998) para implantação do custeio por características são as seguintes: (i) determinar as características dos produtos; (ii) definir as atividades associadas a cada característica do produto; (iii) calcular o custo de cada atividade; (iv) determinar as características dos produtos que causam variação no processo; (v) avaliar o quanto as características dos produtos causam variação no processo; (vi) associar características e parâmetros associados aos produtos; (vii) ajustar os custos das atividades baseados nas características e parâmetros dos produtos. Este artigo fornece uma abordagem diferente da apresentada por Brimson (1998).

Este estudo contribui para literatura na medida em que o modelo de custeio por características proposto não somente é baseado no custeio $A B C$, mas também nas 
unidades de esforço de produção (UEP) e no custo-padrão. Além disso, é apresentada uma aplicação deste modelo em um grupo de poltronas produzidas por uma empresa fabricante de carrocerias de ônibus.

A próxima seção do artigo apresenta uma discussão sobre o conceito de características de produtos. A seção 3 apresenta uma abordagem para o custeio por características propriamente dito e em seguida alguns pré-requisitos para a implementação do modelo são discutidos. Na quinta seção são apresentadas as etapas para a implementação do modelo e na sexta seção é feita a sua aplicação em um grupo de poltronas para ônibus.

\section{DEFINIÇÃO DE CARACTERÍSTICAS DE PRODUTOS}

Para o andamento deste estudo deve-se esclarecer a definição de características, já que na literatura aparecem diversas definições com relação a este termo. Autores da área de custos podem definir o termo de uma forma, no entanto autores de outras áreas do conhecimento podem definir o termo diferentemente. Além de características, ainda serão discutidos outros termos que na literatura podem ter significados similares ao de características como, por exemplo, funções.

Segundo Monden (1999), o motor em um automóvel é uma função, já para Csillag (1995), o motor não é uma função, mas possui a função de fornecer força motora ao automóvel. Quanto ao conceito de característica, para Brimson (1998), o motor é uma característica, confundindo-se, assim, com o conceito de função de Monden (1999). Percebe-se, com isso, o diferente uso dos termos, e por isso recorreuse a autores de outras áreas do conhecimento, não necessariamente de custos, para unificar os conceitos deste trabalho.

Ribeiro et al. (2000) utiliza o desdobramento do produto em partes do produto (PP), característica das partes do produto (CPP) e especificações das partes do produto (ECPP), assim como é apresentado no Quadro 1. Baxter (1998) separa o produto em características funcionais, por exemplo, o cabo, em um descascador de batata, é uma característica funcional, podendo ser redondo ou quadrado. 


\begin{tabular}{|l|l|l|}
\hline \multicolumn{1}{|c|}{ Conceito } & \multicolumn{1}{|c|}{ Definição } & \multicolumn{1}{c|}{ Exemplo (Relacionados a Pneus) } \\
\hline Partes do Produto (PP) & $\begin{array}{l}\text { São as diferentes partes que } \\
\text { compõem produto. }\end{array}$ & Banda, flanco, cintura metálica, lonas. \\
\hline $\begin{array}{l}\text { Característica das Partes do } \\
\text { Produto (CPP) }\end{array}$ & $\begin{array}{l}\text { São as características relacionadas } \\
\text { com as partes dos produtos. }\end{array}$ & $\begin{array}{l}\text { Quanto à banda: largura da banda, } \\
\text { espessura da banda, peso da banda. }\end{array}$ \\
\hline $\begin{array}{l}\text { Especificações das } \\
\text { Características das Partes } \\
\text { do Produto (ECPP) }\end{array}$ & $\begin{array}{l}\text { Especificam as possíveis variações } \\
\text { quanto às características das partes. }\end{array}$ & $\begin{array}{l}\text { Quanto à largura da banda: } 170 \pm 2 \\
\mathrm{~mm}, 201 \pm 2 \mathrm{~mm} \text { ou 226 } \pm 2 \mathrm{~mm} .\end{array}$ \\
\hline
\end{tabular}

Quadro 1 - Conceitos relacionados às definições de características Fonte: RIBEIRO et al., 2000.

Neste artigo adaptou-se o conceito de característica de Brimson (1998) ao modelo de Ribeiro et al. (2000). Para que não sejam confundidas as características das partes do produto (CPP) com as características (features), neste trabalho as características das partes do produto são chamadas de indicadores. Com relação às especificações das partes dos produtos, estas são chamadas somente de especificações. O conceito de características está relacionado com especificações. A Figura 1 mostra a inserção do conceito de características no modelo de Ribeiro et al. (2000).

A estruturação apresentada na Figura 1 foi simplificada, pois se percebeu que esta divisão causa confusão para a equipe envolvida na gestão de custos. O que deve ser então salientado é que esta discussão deve ser feita para o planejamento do produto, mas no que tange a gestão de custos o que interessa são somente as partes do produto e suas características, conforme Figura 2. Por exemplo, o cabo do descascador de batatas é uma parte do produto, e ser redondo ou quadrado diz respeito às suas características. 


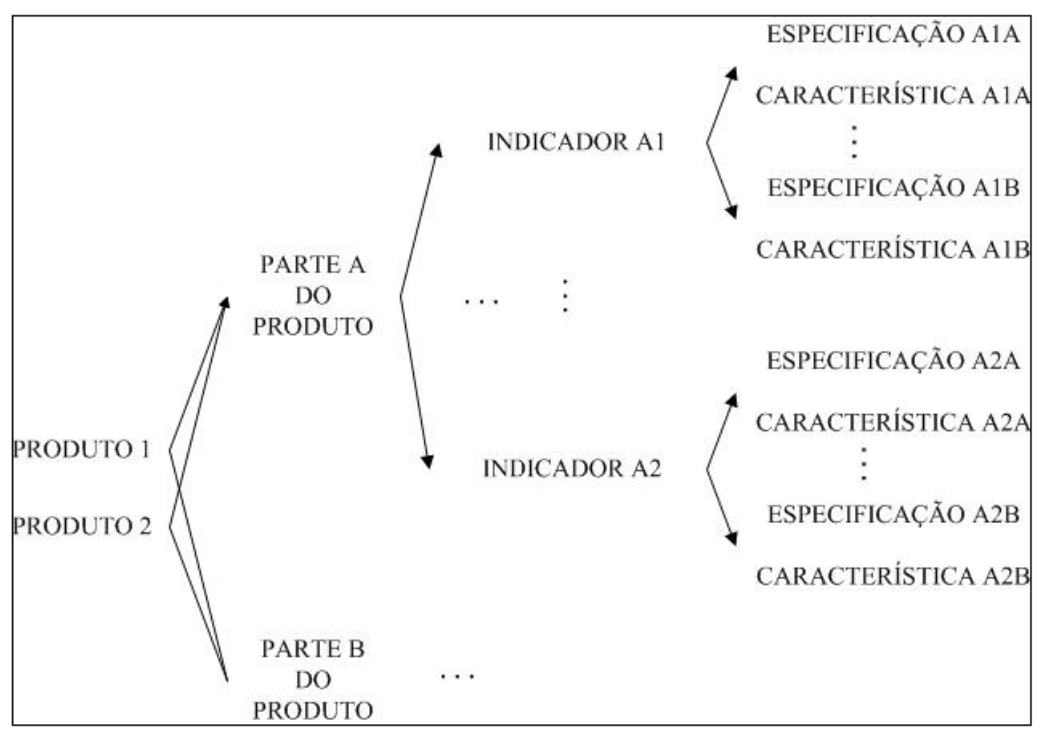

Figura 1 - Desdobramento de partes do produto, indicadores, especificações e características.

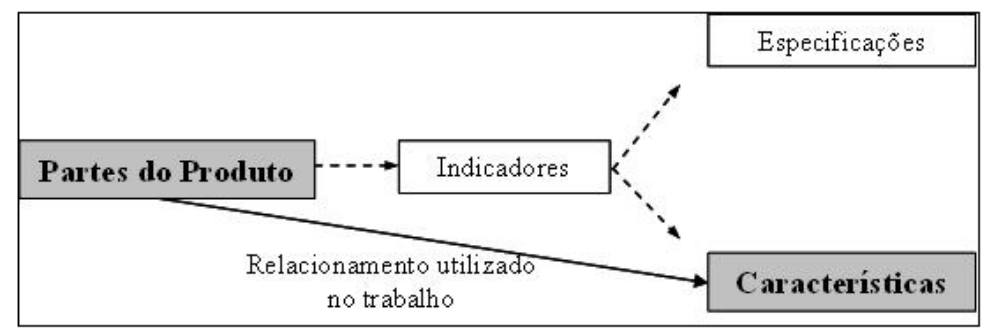

Figura 2 - Desdobramento em partes do produto e características seguidas neste trabalho

Neste artigo os conceitos de partes do produto e características ainda são relacionados aos conceitos de componentes e submontagens do produto. Os componentes e submontagens estão fisicamente representados na árvore do produto, podendo estes ser subdivididos em mais de um nível. Um maior detalhamento a respeito de submontagem e de componentes pode ser encontrado em Elsayed e Boucher (1985). A Figura 3 apresenta uma relação dos conceitos de parte do produto e característica com submontagens e componentes.

Outro conceito introduzido neste estudo é o de elementos comuns. Alguns componentes e submontagens são utilizados independentemente das características, sendo estes chamados de elementos comuns. Os indicadores, que em geral, não são notados, ou são considerados incorporados nos produtos pelos clientes, estão 
relacionadas aos elementos comuns. No que tange à gestão de custos, a definição destes elementos comuns é importante, pois uma redução de seus custos acarreta uma redução de custos no produto, independentemente de sua configuração.

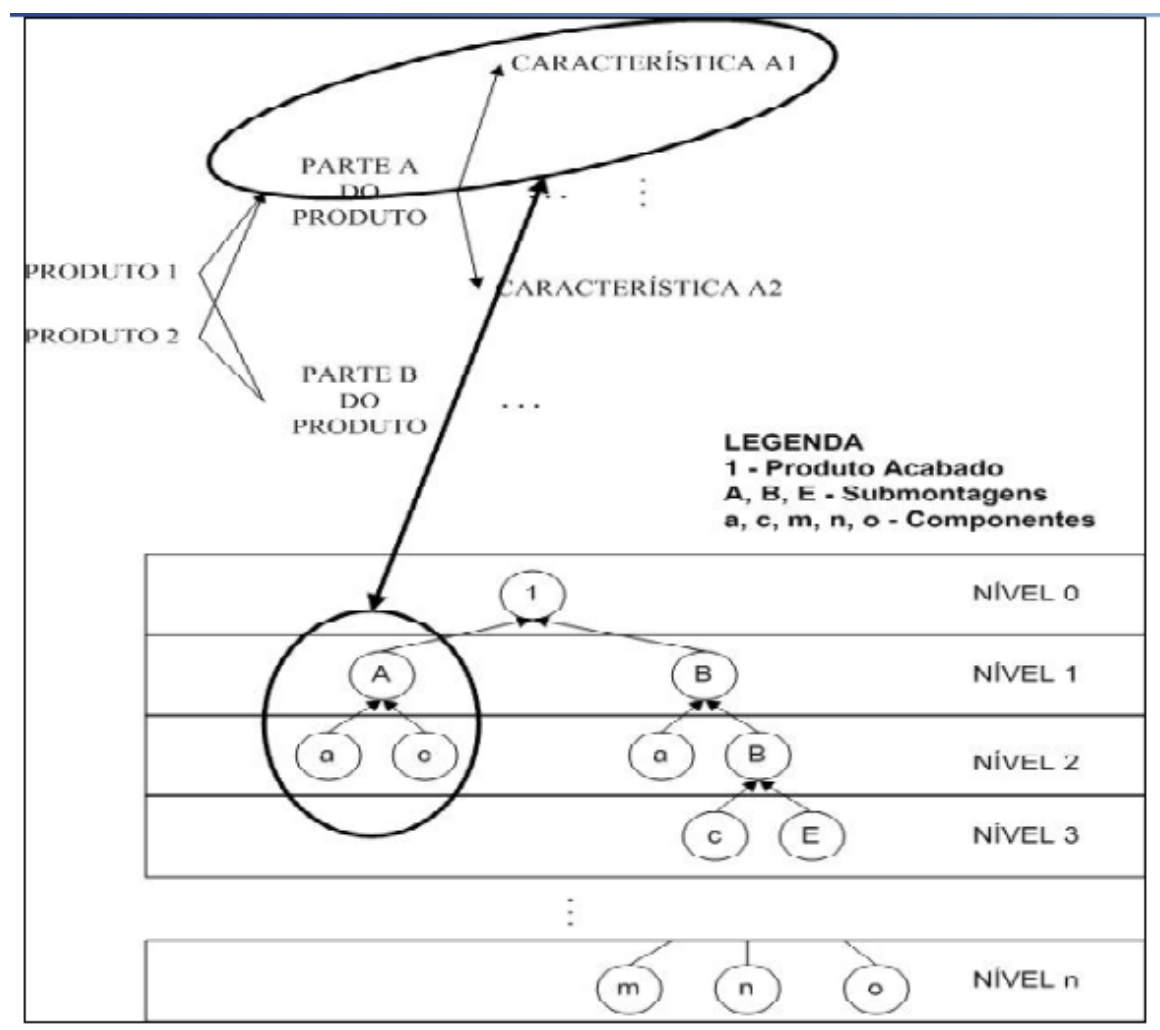

Figura 3 - Equivalência entre parte do produto e característica à submontagens e componentes.

Em termos de cálculo de custos, uma questão que fica é qual a real diferença entre o custeio por características proposto por Brimson (1998) e o que se está propondo neste artigo. Brimson está custeando as características (features) a partir das partes do produto, no entanto ele chama tudo de características (features). Um exemplo que o autor refere em seu artigo é que o motor é uma característica do automóvel subdivida em categorias, como a de ser a diesel ou a gasolina. Nesse estudo o motor seria considerado uma parte do produto, que possuiria a característica de ser a diesel ou a característica de ser a gasolina.

Na prática, tanto Brimson (1998) como os autores desse artigo estão propondo o cálculo do mesmo objeto de custeio, ou seja, as características. No entanto, o 
modelo proposto neste estudo usa além do custeio $A B C$, o método das UEP e o custopadrão. Filomena et al. (2009) apresenta uma discussão semelhante mas para o uso de Custo-Alvo durante o desenvolvimento de produtos. Na próxima seção é discutido o custeio por características propriamente dito.

\section{MODELO DE CUSTEIO POR CARACTERÍSTICAS}

Uma das alterações propostas no modelo de Brimson (1998), além da mudança do conceito de características, diz respeito a como é feita a alocação de custos aos produtos. O método de Brimson é um desdobramento apenas do $A B C$, enquanto o modelo proposto neste artigo se apóia também no trabalho de Kraemer (1995), o qual recomenda a necessidade do uso de mais de um método de custeio para alocação dos custos em empresas, conforme Figura 4. Kraemer (1995), além de se apoiar no método $A B C$ para os custos de apoio, também utiliza o método da UEP para a área industrial, e o custo-padrão para os gastos relativos à matéria-prima.

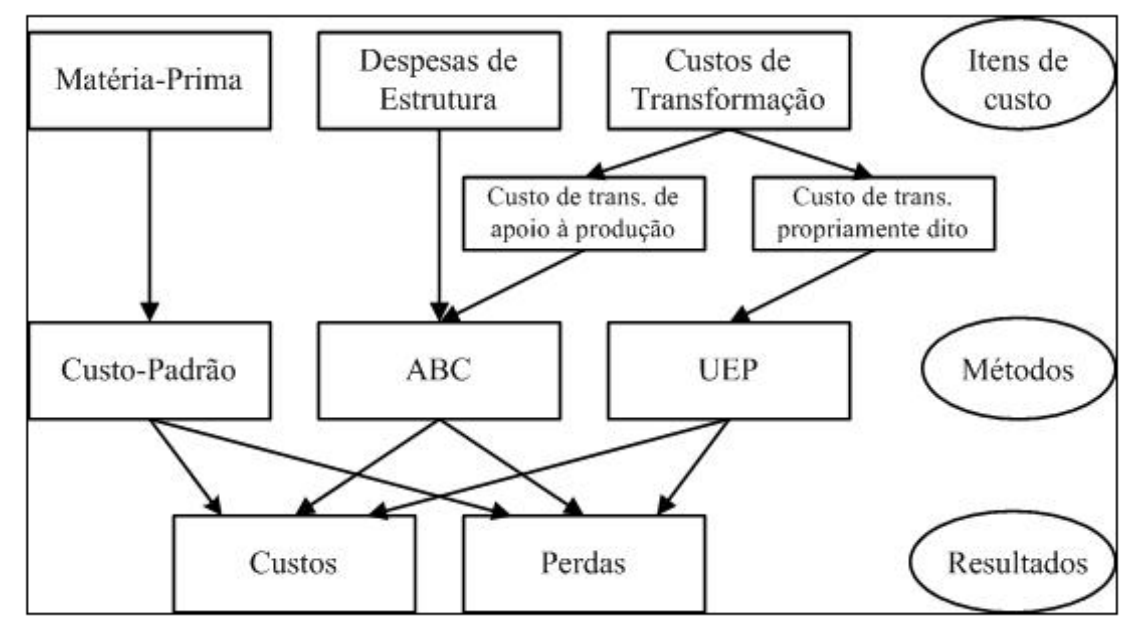

Figura 4 - Combinação de métodos de custeio (KRAEMER, 1995).

Portanto, o método apresentado neste artigo utiliza o desdobramento do produto em partes do produto e características, adaptado do modelo proposto por Brimson (1998) e Ribeiro et al. (2000). Entretanto, a alocação de custos às 
características dos produtos em relação aos itens de custos a serem avaliados, baseia-se no trabalho de Kraemer (1995).

Nem todos os custos podem ser rastreados até as características. Por exemplo, alguns custos provêm de serviços compartilhados, os quais são alocados diretamente aos produtos. Este exemplo também se aplica a alguns processos que indiretamente estão relacionados à fabricação. Como alguns custos não são rastreáveis, deve-se usar alguma base de rateio para a sua distribuição aos produtos.

Quando se analisa o custeio por características, não se está discutindo um novo método de custeio, mas, sim, uma nova forma de alocação de custos aos produtos a partir de um novo objeto de custeio. Isto é verdadeiro já que o cliente, além de adquirir um produto, adquire características a ele associadas, as quais podem variar mesmo dentro de uma mesma categoria de produtos.

Os dados de custos foram classificados, neste trabalho, em três grandes grupos:

a) Custo relativo à matéria-prima: custos relacionados ao consumo de matéria-prima.

b) Custo de transformação: são todos os custos produtivos do produto.

c) Custo de transformação propriamente dito: custos produtivos que agregam valor ao produto.

d) Custo de transformação de apoio à produção: custos de transformação que não agregam valor diretamente aos produtos.

e) Despesa de estrutura: custos administrativos relacionados à produção do produto.

f) A discussão relacionada ao custeio por características, neste artigo, é baseada em três métodos de custeio: o $A B C$, para gastos indiretos de transformação e despesas de estrutura; a UEP, para gastos diretos de transformação; e o custo-padrão, para o cálculo dos gastos de matéria-prima, conforme Kraemer (1995). A Figura 5 apresenta o modelo de sistema de custeio proposto neste artigo. 


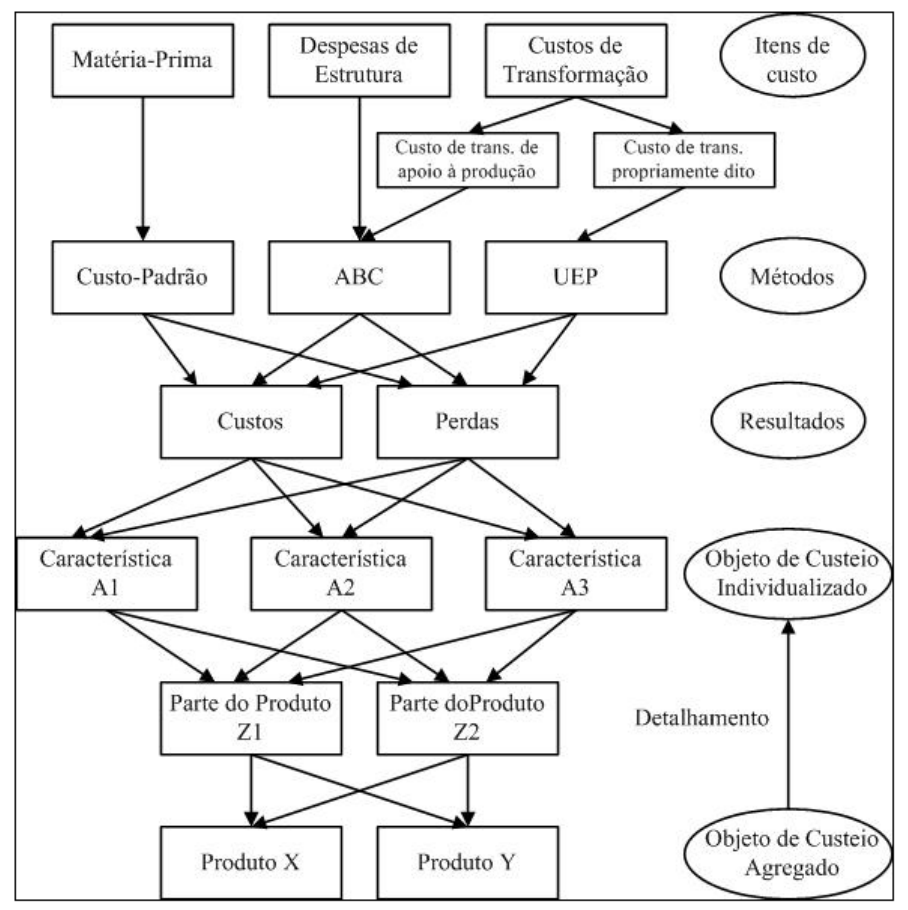

Figura 5 - Método de custeio por características utilizado neste trabalho

Alguns pré-requisitos devem ser atendidos antes da implementação do sistema de custeio por características. Estes são discutidos na próxima seção.

\section{PRÉ-REQUISITOS PARA IMPLANTAÇÃO DO CUSTEIO POR CARACTERÍSTICAS}

Os pré-requisitos para a implementação do sistema de custeio por características passam pela existência de métodos de custeio adequados: custo padrão, UEP e ABC. A seguir são definidos como estes métodos são utilizados para determinação dos: (i) custos de matéria-prima; (ii) custos de transformação (exceto os de apoio à produção); (iii) custos de transformação de apoio à produção e das despesas de estrutura.

\subsection{Determinação dos custos de matéria-prima}

O método do custo-padrão é utilizado para o cálculo do custo de matéria-prima do produto. O cálculo do custo de matéria-prima é relativamente simples. Deve-se 
estabelecer o quanto o objeto de custeio consome de matéria-prima, sendo incluídas neste consumo também as perdas normais relacionadas ao processo. Com a determinação do consumo unitário de matéria-prima por objeto de custeio, basta uma simples multiplicação pela quantidade fabricada do objeto de custeio para se obter a quantidade total consumida de matéria-prima.

\subsection{Determinação dos custos de transformação (exceto os de apoio à produção)}

O método da UEP é utilizado para o cálculo dos custos diretos de transformação do objeto de custeio. Bornia (2002), Kraemer (1995) e Müller (1996) apresentam os passos para sua implantação. A UEP propicia verificar o custo dos produtos no processo produtivo da empresa a partir das suas partes e características. Com o uso da UEP, se prevê os custos relacionados ao processo de chão-de-fábrica, no qual o objeto de custeio está envolvido.

\subsection{Determinação dos custos de transformação de apoio à produção e das despesas de estrutura}

Para o cálculo dos custos de transformação de apoio à produção e das despesas de estrutura é utilizado o custeio baseado em atividades. Nakagawa (1994), Bornia (2002) e Brimson (1996) descrevem as etapas para sua implantação. Com o $A B C$, pretende-se verificar os custos indiretamente envolvidos com a produção relativos às características. $O$ presente estudo não pretende detalhar a implantação do método do custo-padrão, do ABC e da UEP.

\section{ETAPAS PARA IMPLANTAÇÃO DO CUSTEIO POR CARACTERÍSTICAS}

Nesta seção é descrita a arquitetura das informações necessárias para que seja possível a utilização do custeio por características, tendo como base os três métodos de custeio propostos (ABC, UEP e Custo-Padrão). 


\subsection{Determinação das características dos produtos}

Toda a definição das características foi apresentada na seção 2, mas deve-se ressaltar que os objetos de custeio são as partes do produto e suas características.

\subsection{Relacionamento das características e dos elementos comuns aos métodos de custeio}

Primeiramente, deve-se verificar o desdobramento por características feito na etapa 1 (seção 5.1) e, a partir desta fazer a correlação com os métodos de custeio. Ou seja, os objetos de custeio relacionados com os métodos são as características e os elementos comuns. Devem-se criar padrões de relacionamento do objeto de custeio com os métodos de custeio. Esses padrões não são padrões monetários, mas, sim, de consumo. Isso faz com que uma variação no custo da atividade ou do processo atualize automaticamente o valor monetário do objeto de custeio.

\subsubsection{Relacionamento com os custos de matéria-prima}

É simples o relacionamento das características e dos elementos comuns com o método do custo-padrão, uma vez que é uma relação matricial entre o objeto de custeio e o seu consumo de matéria-prima, incluindo as perdas normais do processo.

\subsubsection{Relacionamento com os custos de transformação (exceto os de apoio à produção)}

O cálculo do custo pelo método da UEP só difere na medida em que o objeto de custeio são as características e os elementos comuns, ao invés do produto. Para que se operacionalize o método da UEP é necessário, também, que se saiba o tempo de passagem de cada característica e elemento comum nos postos operativos. Com isso é possível executar a multiplicação dos potenciais produtivos dos postos pelos tempos de passagem.

Para a utilização do método, deve ser adotada uma configuração-base, que deve possuir os mesmos atributos do produto-base, ou seja, representar a estrutura dos processos que as características e os elementos comuns tendem a consumir. Como as características, em geral, consomem os mesmos processos, porém em 
diferentes quantidades, aconselha-se utilizar como configuração-base as características mais utilizadas pelos produtos. Com essas alterações no método, 0 procedimento para o cálculo do custo das partes e características passa a ser o mesmo que para o de produtos.

\subsubsection{Relacionamento com os custos de transformação de apoio à produção e despesas de estrutura}

O custeio baseado em atividades, já mencionado anteriormente, é utilizado para o cálculo dos custos de transformação de apoio e das despesas de estrutura. As duas primeiras etapas para a implementação do método continuam iguais, ou seja, devem ser mapeadas as atividades e, em seguida, alocados os custos às atividades com base nos direcionadores de recursos.

A diferença está, assim como no método da UEP, na alocação do consumo das atividades pelos objetos de custeio. Deve se relacionar os direcionadores das atividades às características e aos elementos comuns, e não aos produtos. Esse detalhamento é fundamental para algumas áreas de apoio como, por exemplo, a engenharia, já que as características do produto estão diretamente ligadas às atividades de re-projeto de produto, de desenvolvimento dos fornecedores, entre outras.

Nem todos os custos indiretos necessitam do detalhamento do produto. Logo, estes poderão ser alocados diretamente aos produtos, sendo considerados como serviços compartilhados. Um exemplo é a área de vendas, já que para essa o detalhamento do objeto de custeio de produto para partes ou características, não acarretaria numa melhor alocação de custos aos produtos.

\subsection{Determinar o custo das características e dos elementos comuns}

Por meio do relacionamento das características e dos elementos comuns com os métodos de custeio, é possível calcular os custos dos objetos de custeio. Para isso, é proposta uma simples álgebra matricial, na qual as linhas são os objetos de custeio e as colunas as atividades, operações ou matérias-prima. No cruzamento entre 
colunas e linhas, e com base no consumo determinado na etapa 2 (seção 5.2), podese calcular o custo das características e dos elementos comuns.

\subsection{Formar o custo do produto a partir de suas características e elementos comuns}

O custo do produto, no custeio baseado em características, deve ser formado a partir das características e dos elementos comuns, ou seja, é um novo nível de rastreabilidade de custos. Deve se determinar, então, as características que são consumidas pelos produtos. A Figura 5 apresenta os relacionamentos entre custos, métodos de custeio e objetos de custeio propostos neste método.

\section{CUSTEIO POR CARACTERÍSTICAS - APLICAÇÃO}

A aplicação do modelo segue as etapas e subdivisões definidas na seção anterior. O estudo apresenta o custeio de um grupo de poltronas produzidas por uma empresa fabricante de carrocerias de ônibus.

\subsection{Determinação das características do produto}

Inicialmente, para exemplificar a utilização dos indicadores e especificações, é efetuado o desdobramento da poltrona em partes do produto, indicadores, especificações e características. As partes do produto foram divididas: em parte estrutural e parte de acabamento, e seu desdobramento até características é apresentado na Figura 6.

Para a parte estrutural do produto foi definido um indicador único: índice de mobilidade ao levantar e existência de cinzeiro, já que um influencia diretamente o outro. O índice de mobilidade ao levantar se refere à facilidade de locomoção propiciada pela especificação de $0^{\circ}$ de mobilidade e de $0^{\circ}$ a $120^{\circ}$ de mobilidade tendo, ainda, concatenado a este indicador a existência ou não de cinzeiro. Para cada especificação, tanto de mobilidade como de existência ou não de cinzeiro, está associada uma característica. 
O tipo de anatomia e de revestimento, assim como a existência de porta revista, foram os indicadores definidos para a parte do produto relacionada ao acabamento. $\mathrm{O}$ tipo de anatomia está relacionado com o formato da espuma a ser utilizada, sendo especificada como anatomia convencional ou anatomia soft, estando ainda associado a este indicador o tipo de revestimento, que pode ser especificado como tecido ou vulcouro. Outro indicador seria a existência, ou não, de porta-revista, a qual está associada à facilidade de armazenar material literário. Também, para cada especificação de acabamento está associada uma característica.

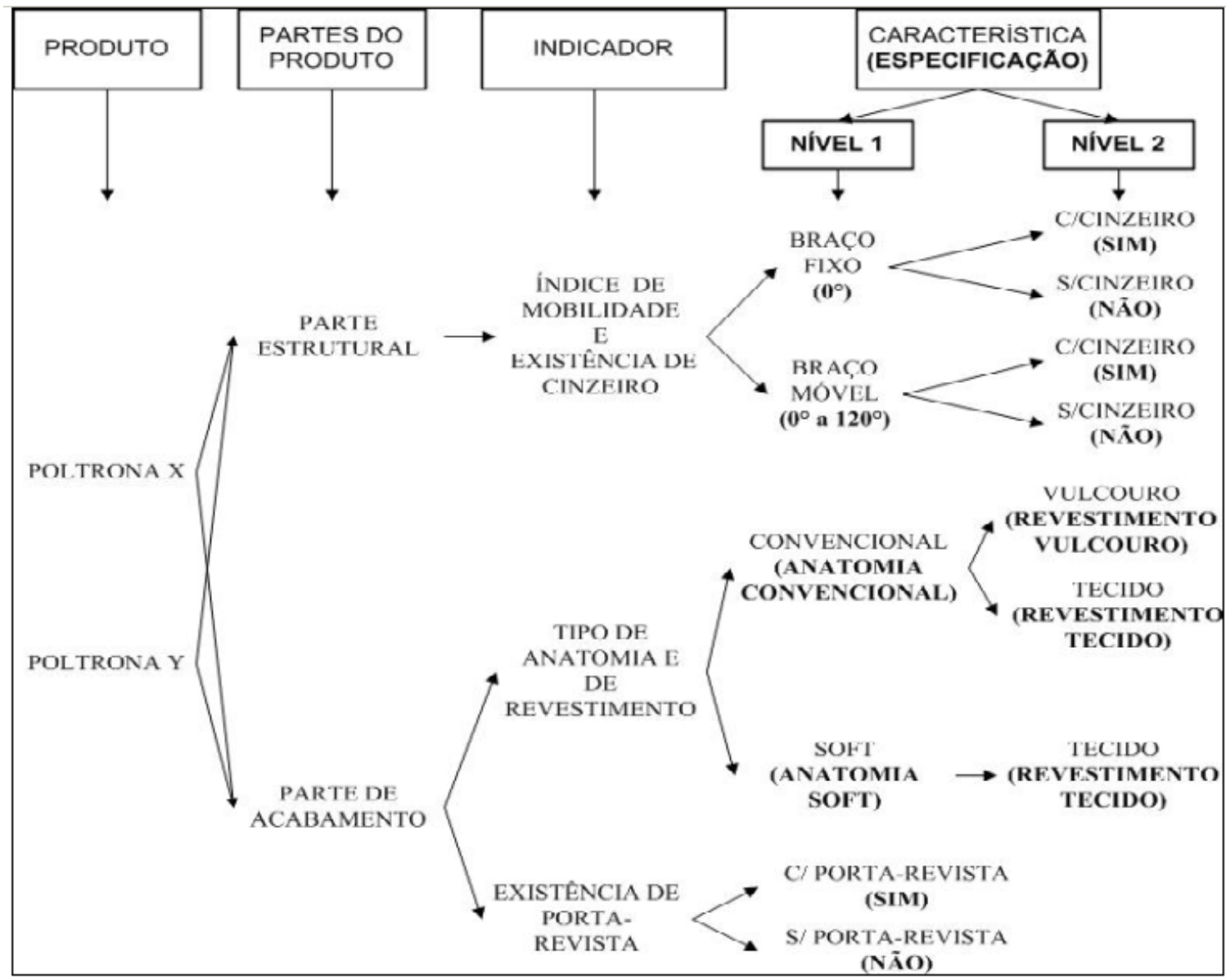

Figura 6 - Desdobramento da poltrona incluindo os indicadores e especificações 
Não se procurou detalhar todos os indicadores, mas apenas aqueles que pareceram demandar diferentes esforços no processo produtivo. Como foi apresentado no modelo, os indicadores e as especificações não são detalhados durante o custeio das características, sendo, então, efetuado o relacionamento direto das partes do produto com as características, conforme a Figura 7.

Após a definição das partes do produto e das características, devem-se relacionar ambas às submontagens e aos componentes do produto, sendo que algumas submontagens e alguns componentes podem ser os mesmos independentemente da característica do produto, como já descrito no modelo, ou seja, elementos comuns. O Quadro 2 apresenta algumas submontagens e componentes da parte estrutural da poltrona que são utilizadas em todas as características, ou seja, tanto nas poltronas com braço fixo como nas poltronas com braço móvel. Tais submontagens e componentes são denominados de elementos estruturais comuns.

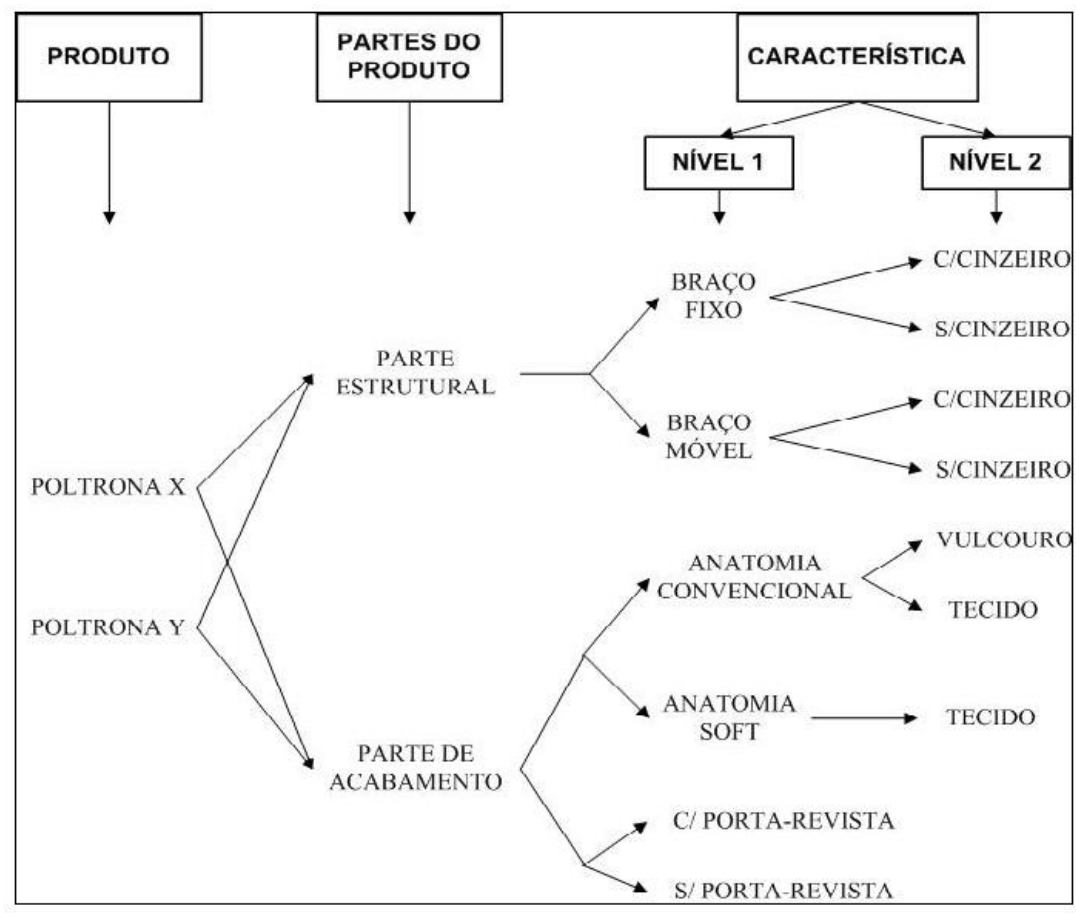

Figura 7 - Desdobramento da poltrona excluindo os indicadores e as especificações 


\begin{tabular}{|c|c|c|}
\hline & Código & Descrição \\
\hline \multirow{8}{*}{ 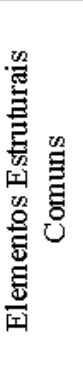 } & 32953 & Bracelete Central \\
\hline & 2657 & Bucha Articulacao Braco Central P \\
\hline & 3374 & Paraf.Sext. $7 / 16^{\prime \prime X} 7 / 8 "$ 14unc \\
\hline & 2481 & Arruela Poliamida Braco Central M \\
\hline & 32976 & Limitador \\
\hline & 1175 & Tint.Potx Prt Poliester \\
\hline & 473 & Paraf. PH.N.2 CO ZP 4,2X25,0 \\
\hline & 72812 & Bracalete Fx.S/Cinz.Pant. $648 \mathrm{u}$ Cp0 \\
\hline
\end{tabular}

Quadro 2 - Elementos comuns para a parte estrutural da poltrona

Assim como foram definidos elementos comuns para a parte estrutural da poltrona, mais especificamente para a existência de braço fixo ou móvel, pode-se definir elementos comuns para as diferentes partes e características. O Quadro 3 apresenta os elementos comuns e características que foram relacionados para cada uma das partes do produto.

\begin{tabular}{|c|c|c|c|}
\hline $\begin{array}{l}\text { Parte do } \\
\text { Produto }\end{array}$ & \multicolumn{3}{|c|}{ Características } \\
\hline \multirow{7}{*}{ 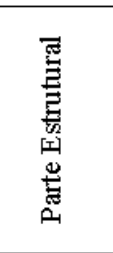 } & \multicolumn{3}{|c|}{ (2) Elementos Estruturais Comuns } \\
\hline & \multirow{3}{*}{ Braço Fixo } & \multicolumn{2}{|c|}{ (21) Elementos Fixos Comuns } \\
\hline & & \multirow{2}{*}{ Cinzeiro } & (2ab1) $\mathrm{C} /$ Cinzeiro \\
\hline & & & (2ab2) S/Cinzeiro \\
\hline & \multirow{3}{*}{ Braço Móvel } & \multicolumn{2}{|c|}{ (22) Elementos Móveis Comuns } \\
\hline & & \multirow{2}{*}{ Cinzeiro } & (2bb1) $\mathrm{C} /$ Cinzeiro \\
\hline & & & (2bb2) S/Cinzeiro \\
\hline \multirow{7}{*}{ 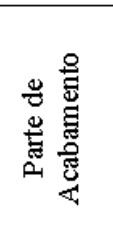 } & \multirow{3}{*}{ Convencional } & \multicolumn{2}{|c|}{ (31) Elementos Convencionais Comuns } \\
\hline & & \multirow{2}{*}{ Revestimento } & (3a1) Vulcouro \\
\hline & & & (3a2) Tecido \\
\hline & \multirow{2}{*}{ Soft } & \multicolumn{2}{|c|}{ (32) Elementos Soft Comuns } \\
\hline & & Revestimento & (3b) Tecido \\
\hline & \multirow{2}{*}{ Porta-Revista } & \multicolumn{2}{|c|}{ (4a) C/Porta-Revista } \\
\hline & & \multicolumn{2}{|c|}{ (4b) S/Porta-Revista } \\
\hline
\end{tabular}

Quadro 3 - Definição dos elementos comuns das características e das partes

Definiram-se elementos comuns para as características relacionadas tanto à utilização de braço fixo como de braço móvel. Também, relacionaram-se elementos 
comuns para anatomia convencional e para anatomia soft. Para o porta-revista optouse em não se definir elementos comuns, pois como foi descrito na seção 2.1 em alguns casos eles podem não ser explicitados. Neste caso, eles não foram particularizados devido ao seu baixo valor monetário.

Para facilitar o desenvolvimento do estudo, colocou-se um código ao lado de cada característica para permitir a sua aglutinação. Por exemplo, caso se quisesse uma poltrona com Braço Fixo, Sem Cinzeiro, Anatomia Convencional, Revestimento de Tecido, e com Porta-Revista ter-se-ia a seguinte codificação: $(2)+(21)+(2 a b 2)+$ $(31)+(3 a 2)+(4 a)$.

Com a definição das partes do produto e das características, pôde-se partir para a determinação dos custos.

\subsection{Relacionar as características e os elementos comuns aos métodos de custeio}

Nesta fase são feitos os relacionamentos das características e elementos comuns com os métodos de custeio. Ou seja, esses dois passam a ser os objetos de custeio do sistema. A relação entre as características e elementos comuns é feita através das submontagens e componentes que as formam.

\subsubsection{Relacionamento com os custos de matéria-prima}

O custo de matéria-prima é relativamente simples de ser avaliado, na medida que para isso é necessário somente fazer o levantamento dos componentes que são utilizados para composição do produto. A Tabela 1 apresenta o custo relativo à matéria-prima com relação às características e elementos comuns. 
Tabela 1 - Custo relativo à matéria-prima das características e elementos comuns da poltrona

\begin{tabular}{|c|c|c|c|c|}
\hline $\begin{array}{l}\text { Parte do } \\
\text { Produto }\end{array}$ & \multicolumn{3}{|c|}{ Características } & Custo MP \\
\hline \multirow{7}{*}{ 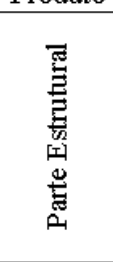 } & \multicolumn{3}{|c|}{ (2) Elementos Estruturais Comuns } & $\mathrm{R} \$ 78,74$ \\
\hline & \multirow{3}{*}{ Braço Fixo } & \multicolumn{2}{|c|}{ (21) Elementos Fixos Comuns } & - \\
\hline & & \multirow{2}{*}{ Cinzeiro } & (2ab1) C/Cinzeiro & $\mathrm{R} \$ 14,06$ \\
\hline & & & (2ab2) S/Cinzeiro & $\mathrm{R} \$ 11,65$ \\
\hline & \multirow{3}{*}{ Braço Móvel } & \multicolumn{2}{|c|}{ (22) Elementos Móveis Comuns } & $\mathrm{R} \$ 3,40$ \\
\hline & & \multirow{2}{*}{ Cinzeiro } & (2bb1) $\mathrm{C} /$ Cinzeiro & $\mathrm{R} \$ 21,09$ \\
\hline & & & (2bb2) S/Cinzeiro & $\mathrm{R} \$ 19,82$ \\
\hline \multirow{7}{*}{ 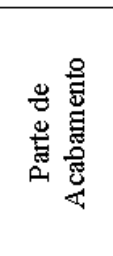 } & \multirow{3}{*}{ Convencional } & \multicolumn{2}{|c|}{ (31) Elementos Convencionais Comuns } & $\mathrm{R} \$ 67,75$ \\
\hline & & \multirow{2}{*}{ Revestimento } & (3a1) Vulcouro & $\mathrm{R} \$ 27,03$ \\
\hline & & & (3a2) Tecido & $\mathrm{R} \$ 67,61$ \\
\hline & \multirow{2}{*}{ Soft } & \multicolumn{2}{|c|}{ (32) Elementos Soft Comuns } & $\mathrm{R} \$ 102,28$ \\
\hline & & Revestimento & (3b) Tecido & $\mathrm{R} \$ 79,62$ \\
\hline & \multirow{2}{*}{ Porta-Revista } & \multicolumn{2}{|c|}{ (4a) C/Porta-Revista } & $\mathrm{R} \$ 13,55$ \\
\hline & & \multicolumn{2}{|c|}{ (4b) S/Porta-Revista } & $\mathrm{R} \$ 11,09$ \\
\hline
\end{tabular}

\subsubsection{Relacionamento com os custos de transformação (exceto os de apoio à produção)}

Com o valor dos potenciais produtivos dos postos operativos basta multiplicar o tempo de passagem das características e elementos comuns pelos postos e, com isso, obtém-se o valor dessas em UEPs, conforme Tabela 2.

Com base num valor de $\mathrm{R} \$ 35,00$ por UEP, foi calculado o valor do custo de transformação das características e elementos comuns. A Tabela 3 mostra o valor do custo de transformação das características e elementos comuns em reais.

\subsubsection{Relacionamentos com os custos de transformação de apoio à produção e despesas de estrutura}

A empresa não permitiu, por questões estratégicas, a elaboração do custeio ABC na sua estrutura. No entanto, para que fossem considerados os custos indiretos relativos às características, fez-se um estudo com base nos custos diretos e indiretos da empresa. Com isso, percebeu-se que os custos indiretos representavam $35 \%$ dos custos diretos. A Tabela 4 apresenta os custos de apoio à produção e despesas de estrutura, que foram obtidos pela multiplicação dos dados da Tabela 3 por 35\%. 
Tabela 2 - Valor das características e elementos em UEP's

\begin{tabular}{|c|c|c|c|c|}
\hline $\begin{array}{l}\text { Parte do } \\
\text { Produto }\end{array}$ & \multicolumn{3}{|c|}{ Características } & UEP \\
\hline \multirow{7}{*}{ 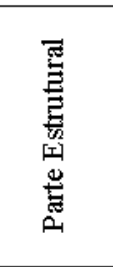 } & \multicolumn{3}{|c|}{ (2) Elementos Estruturais Comuns } & 0,458 \\
\hline & \multirow{3}{*}{ Braço Fixo } & \multicolumn{2}{|c|}{ (21) Elementos Fixos Comuns } & 0,258 \\
\hline & & \multirow{2}{*}{ Cinzeiro } & (2ab1) $\mathrm{C} /$ Cinzeiro & - \\
\hline & & & (2ab2) $\mathrm{S} /$ Cinzeiro & - \\
\hline & \multirow{3}{*}{ Braço Móvel } & \multicolumn{2}{|c|}{ (22) Elementos Móveis Comuns } & 0,274 \\
\hline & & & (2bb1) $\mathrm{C} /$ Cinzeiro & - \\
\hline & & & (2bb2) S/Cinzeiro & - \\
\hline \multirow{7}{*}{ 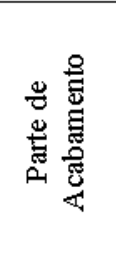 } & \multirow{3}{*}{ Convencional } & \multicolumn{2}{|c|}{ (31) Elementos Convencionais Comuns } & 0,060 \\
\hline & & \multirow{2}{*}{ Revestimento } & (3a1) Vulcouro & 0,248 \\
\hline & & & (3a2) Tecido & 0,187 \\
\hline & \multirow{2}{*}{ Soft } & \multicolumn{2}{|c|}{ (32) Elementos Soft Comuns } & 0,060 \\
\hline & & Revestimento & (3b) Tecido & 0,218 \\
\hline & \multirow{2}{*}{ Porta-Revista } & \multicolumn{2}{|c|}{ (4a) C/Porta-Revista } & 0,036 \\
\hline & & \multicolumn{2}{|c|}{ (4b) S/Porta-Revista } & 0,017 \\
\hline
\end{tabular}

Tabela 3 - Valor do custo direto de transformação das características e elementos comuns em reais

\begin{tabular}{|c|c|c|c|c|}
\hline $\begin{array}{l}\text { Parte do } \\
\text { Produto }\end{array}$ & \multicolumn{3}{|c|}{ Características } & $\begin{array}{l}\text { Custo Diretos de } \\
\text { Transformacâáo }\end{array}$ \\
\hline \multirow{7}{*}{ 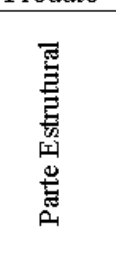 } & \multicolumn{3}{|c|}{ (2) Elementos Estruturais Comuns } & $\mathrm{R} \$ 16,04$ \\
\hline & \multirow{3}{*}{ Braço Fixo } & \multicolumn{2}{|c|}{ (21) Elementos Fixos Comuns } & $\mathrm{R} \$ 9,01$ \\
\hline & & \multirow{2}{*}{ Cinzeiro } & (2ab1) $\mathrm{C} /$ Cinzeiro & - \\
\hline & & & (2ab2) S/Cinzeiro & - \\
\hline & \multirow{3}{*}{ Braço Móvel } & \multicolumn{2}{|c|}{ (22) Elementos Móveis Comuns } & $\mathrm{R} \$ 9,58$ \\
\hline & & \multirow{2}{*}{ Cinzeiro } & (2bb1) $\mathrm{C} /$ Cinzeiro & - \\
\hline & & & (2bb2) S/Cinzeiro & - \\
\hline \multirow{7}{*}{ 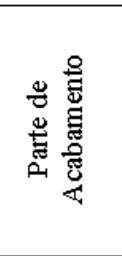 } & \multirow{3}{*}{ Convencional } & \multicolumn{2}{|c|}{ (31) Elementos Convencionais Comuns } & $\mathrm{R} \$ 2,11$ \\
\hline & & \multirow{2}{*}{ Revestimento } & (3a1) Vulcouro & $\mathrm{R} \$ 8,68$ \\
\hline & & & (3a2) Tecido & $\mathrm{R} \$ 6,56$ \\
\hline & \multirow{2}{*}{ Soft } & \multicolumn{2}{|c|}{ (32) Elementos Soft Comuns } & $\mathrm{R} \$ 2,11$ \\
\hline & & Revestimento & (3b) Tecido & $\mathrm{R} \$ 7,64$ \\
\hline & \multirow{2}{*}{ Porta-Revista } & \multicolumn{2}{|c|}{ (4a) C/Porta-Revista } & $\mathrm{R} \$ 1,28$ \\
\hline & & \multicolumn{2}{|c|}{ (4b) S/Porta-Revista } & $\mathrm{R} \$ 0,61$ \\
\hline
\end{tabular}

\subsection{Determinar o custo das características e elementos comuns}

Após o cálculo dos custos de matéria-prima, diretos de transformação, de apoio à transformação e despesas de estrutura, faz-se a consolidação dos mesmos, conforme a Tabela 5. 


\subsection{Determinar o custo do produto a partir de suas características e elementos comuns}

Com a determinação do custo das características e elementos comuns, pôde-se calcular o custo dos produtos. Um exemplo é a Poltrona Convencional de Tecido, Bracelete Fixo, Sem Cinzeiro e Porta Revista (Configuração A) tendo a seguinte configuração: $(2)+(21)+(2 a b 2)+(31)+(3 a 2)+(4 a)$. O custo estimado total desta poltrona é demonstrado na Equação (1). Para o cálculo da equação se utilizou como base os dados da Tabela 5 .

Custo Configuração $A=100,40+12,17+11,65+70,59+76,47+15,27=R \$ 286,55$

Tabela 4 - Valor dos custos indireto de transformação e despesas de estrutura das características e elementos comuns em reais

\begin{tabular}{|c|c|c|c|c|}
\hline $\begin{array}{l}\text { Parte do } \\
\text { Produto }\end{array}$ & \multicolumn{3}{|c|}{ Características } & $\begin{array}{c}\text { Custo } \\
\text { Indiretos }\end{array}$ \\
\hline \multirow{7}{*}{ 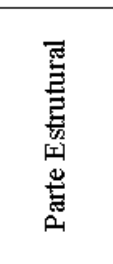 } & \multicolumn{3}{|c|}{ (2) Elementos Estruturais Comuns } & $\mathrm{R} \$ 5,62$ \\
\hline & \multirow{3}{*}{ Braço Fixo } & \multicolumn{2}{|c|}{ (21) Elementos Fixos Comuns } & $\mathrm{R} \$ 3,15$ \\
\hline & & \multirow{2}{*}{ Cinzeiro } & (2ab1) $\mathrm{C} /$ Cinzeiro & - \\
\hline & & & (2ab2) $\mathrm{S} /$ Cinzeiro & - \\
\hline & \multirow{3}{*}{ Braço Móvel } & \multicolumn{2}{|c|}{ (22) Elementos Móveis Comuns } & $\mathrm{R} \$ 3,35$ \\
\hline & & \multirow{2}{*}{ Cinzeiro } & (2bb1) C/Cinzeiro & - \\
\hline & & & (2bb2) S/Cinzeiro & - \\
\hline \multirow{7}{*}{ 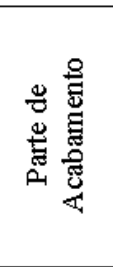 } & \multirow{3}{*}{ Convencional } & \multicolumn{2}{|c|}{ (31) Elementos Convencionais Comuns } & $\mathrm{R} \$ 0,74$ \\
\hline & & \multirow{2}{*}{ Revestimento } & (3a1) Vulcouro & $\mathrm{R} \$ 3,04$ \\
\hline & & & (3a2) Tecido & $\mathrm{R} \$ 2,30$ \\
\hline & \multirow{2}{*}{ Soft } & \multicolumn{2}{|c|}{ (32) Elementos Soft Comuns } & $\mathrm{R} \$ 0,74$ \\
\hline & & Revestimento & (3b) Tecido & $\mathrm{R} \$ 2,67$ \\
\hline & \multirow{2}{*}{ Porta-Revista } & \multicolumn{2}{|c|}{ (4a) C/Porta-Revista } & $\mathrm{R} \$ 0,45$ \\
\hline & & \multicolumn{2}{|c|}{ (4b) S/Porta-Revista } & $\mathrm{R} \$ 0,21$ \\
\hline
\end{tabular}


Tabela 5 - Cálculo do custo do produto - custos de matéria-prima (MP), custos diretos de transformação $(\mathrm{CD})$ e custos de apoio à transformação e despesas de estrutura $(\mathrm{Cl})$

\begin{tabular}{|c|c|c|c|c|c|c|c|}
\hline P.P & \multicolumn{3}{|c|}{ Características } & $\mathrm{MP}(\mathrm{R} \$)$ & $\mathrm{CD}(\mathrm{R} \$)$ & $\begin{array}{c}\mathrm{CI} \\
(\mathrm{R} \$)\end{array}$ & $\begin{array}{l}\text { Custo } \\
\text { Total } \\
\text { (R\$) }\end{array}$ \\
\hline \multirow{7}{*}{ 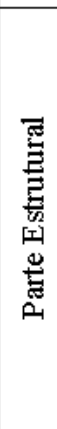 } & \multicolumn{3}{|c|}{ (2) Elementos Estruturais Comuns } & 78,74 & 16,04 & 5,62 & 100,40 \\
\hline & \multirow{3}{*}{ Braço Fixo } & \multicolumn{2}{|c|}{ (21) Elementos Fixos Comuns } & - & 9,01 & 3,15 & 12,17 \\
\hline & & \multirow{2}{*}{ Cinzeiro } & \begin{tabular}{|l|} 
(2ab1) \\
C/Cinzeiro
\end{tabular} & 14,06 & - & - & 14,06 \\
\hline & & & \begin{tabular}{|l|} 
(2ab2) \\
S/Cinzeiro \\
\end{tabular} & 11,65 & - & - & 11,65 \\
\hline & \multirow{3}{*}{ Braço Móvel } & \multicolumn{2}{|c|}{ (22) Elementos Móveis Comuns } & 3,40 & 9,58 & 3,35 & 16,34 \\
\hline & & \multirow{2}{*}{ Cinzeiro } & \begin{tabular}{|l|} 
(2bb1) \\
C/Cinzeiro
\end{tabular} & 21,09 & - & - & 21,09 \\
\hline & & & \begin{tabular}{|l|} 
(2bb2) \\
S/Cinzeiro
\end{tabular} & 19,82 & - & - & 19,82 \\
\hline \multirow{7}{*}{ 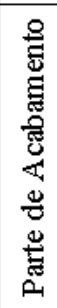 } & \multirow{3}{*}{$\begin{array}{l}\text { Convenciona } \\
1\end{array}$} & \multicolumn{2}{|c|}{$\begin{array}{l}\text { (31) Elementos Convencionais } \\
\text { Comuns }\end{array}$} & 67,75 & 2,11 & 0,74 & 70,59 \\
\hline & & \multirow{2}{*}{ Revestimento } & $\left(3^{\mathrm{a}} 1\right)$ Vulcouro & 27,03 & 8,68 & 3,04 & 38,75 \\
\hline & & & $\left(3^{a} 2\right)$ Tecido & 67,61 & 6,56 & 2,30 & 76,47 \\
\hline & \multirow{2}{*}{ Soft } & \multicolumn{2}{|c|}{ (32) Elementos Soft Comuns } & 102,28 & 2,11 & 0,74 & 105,12 \\
\hline & & Revestimento & (3b) Tecido & 79,62 & 7,64 & 2,67 & 89,94 \\
\hline & \multirow{2}{*}{\begin{tabular}{|l|} 
Porta- \\
Revista
\end{tabular}} & \multicolumn{2}{|c|}{ (4a) C/Porta-Revista } & 13,55 & 1,28 & 0,45 & 15,27 \\
\hline & & \multicolumn{2}{|c|}{ (4b) S/Porta-Revista } & 11,09 & 0,61 & 0,21 & 11,91 \\
\hline
\end{tabular}

\section{CONSIDERAÇÕES FINAIS}

Este artigo apresentou um modelo estruturado para a utilização do custeio por características. Nele foram discutidos o conceito de características, custeio por características, pré-requisitos para implantação do modelo e as etapas para implantação do modelo. Após, foi apresentada uma aplicação do modelo em uma indústria fabricante de carrocerias de ônibus.

A gestão de custo por características se mostra importante na medida em que cada vez mais os produtos são compostos por diversas características. Logo, começa a ser imperativo o custeio das características dos produtos para que, após, possa-se estruturar o custo do produto como um todo.

O estudo apresenta algumas limitações como: o não tratamento dos custos indiretos a partir do conceito de características e a não formulação da hipótese da utilização do método dos centros de custos para os custos diretos. Como pesquisas futuras recomenda-se a aplicação do custeio $A B C$ e do conceito de características 
para custos indiretos.

\section{Agradecimentos}

Os autores agradecem ao apoio finaceiro da CAPES para o desenvolvimento deste estudo.

\section{REFERÊNCIAS}

BAXTER, M. (1993). Projeto de produto. São Paulo, Ed. Edgard Blücher. (2 ed.).

BERLINER, C. e BRIMSON, J. A. (1988). Gerenciamento de custos em indústrias avançadas: base conceitual CAM-I. São Paulo, Editora T. A. Queiroz.

BRIMSON, J. A. (1996). Contabilidade por atividades. São Paulo, Ed. Atlas.

BRIMSON, J. A. Feature Costing: Beyond ABC. Journal of Cost Management, Jan./Fev., p. 6-12.

BRIMSON, J.A. (2001). Using Predictive Accounting to Improve Product Management. International Journal of Strategic Cost Management, 2 (3), Summer.

CSILLAG, J. M. (1995). Análise de valor. São Paulo. Ed. Atlas. (4 ed.).

ELSAYED, E. A. e BOUCHER, T. O. (1985). Analysis and Control of Production Systems. New Jersey, Ed. Printice-Hall.

FILOMENA, T.P., KLIEMANN, F.J. e DUFFEY, M. (2009). Target costing operationalization during product development: Model and application. International Journal of Production Economics, doi: 10.1016/j.ijpe.2008.12.007, em fase de impressão.

JOHNSON, H. T. e KAPLAN, R. S. (1993). Contabilidade Gerencial. Rio de Janeiro: Ed. Campus.

KRAEMER, T. H. (1995). Discussão de um Sistema de Custeio Adaptado às Exigências da Nova Competição Global. Porto Alegre, RS. (Dissertação de Mestrado). Programa de Pós-Graduação em Engenharia de produção, Universidade Federal do Rio Grande do Sul - UFRGS.

LEIBL, P., HUNDAL, M. e HOEHNE, G. (1999). Cost Calculation with a Feature-based CAD System using Modules for Calculation. Comparison and Forecast. Journal of Engineering Design, 10(1), p. 93-102. 
MONDEN, Y. (1999). Sistemas de redução de custos: custo-alvo e custo kaizen. Porto Alegre, Ed. Bookman.

MÜLLER, C. J. A. (1996). Evolução dos Sistemas de Manufatura e a Necessidade de Mudança nos Sistemas de Controle e Custeio. Porto Alegre, RS. (Dissertação de Mestrado). Programa de Pós-Graduação em Engenharia de produção, Universidade Federal do Rio Grande do Sul - UFRGS.

NAKAGAWA, M. (1994). ABC - Custeio Baseado em Atividades. São Paulo. Ed. Atlas.

OU-YANG, C. e LIN, T. S. (1997). Developing an Integrated Framework for FeatureBased Early manufacturing Cost Estimation. International Journal of Advanced Manufacturing Tecnology, 13, p. 618-629.

RIBEIRO, J. L. D., ECHEVESTE, M. E. e DANILEVICZ, A. M. (2000). A utilização do QFD na otimização de produtos, processos e serviços. Porto Alegre, Feeng, $2^{\circ}$ Reimpressão.

ROSA, F.S. e LUNKES, R.J. (2006). O Uso do Custom-Made Based Costing-CBC no Custeamento das Características de Produtos e Serviços. ABCustos, 1(1), set.-dez.

Data de Submissão: 05/02/2009

Data de Aceite: 23/04/2009 